\title{
Critique Generation to Increase Diversity in Conversational Recipe Recommender System
}

\author{
Fakhri Abbas ${ }^{1}$, Nadia Najjar ${ }^{2}$ and David Wilson ${ }^{3}$ \\ fabbas1@uncc.edu ${ }^{1}$, nanajjar@uncc.edu ${ }^{2}$, david.Wilson@uncc.edu ${ }^{3}$ \\ Department of Information Systems \\ University of North Carolina at Charlotte \\ Charlotte, NC
}

\begin{abstract}
Conversational recommender systems help to guide users in exploring the search space in order to discover items of interest. During the exploration process, the user provides feedback on recommended items to refine subsequent recommendations. Critiquing as a way of feedback has proven effective for conversational interactions. In addition, diversifying the recommended items during exploration can help to increase user understanding of the search space, which critiquing alone will not achieve. Both aspects are important elements for recommender applications in the food domain. Diversity in diet has been shown to predict nutritional health, and conversational exploration can help to introduce new food items. In this paper, we introduce a novel approach that brings together critique and diversity to support conversational recommendation in the recipe domain. Initial evaluation in comparison to a baseline similarity-based recommender shows that the proposed approach increases diversity during the exploration process.
\end{abstract}

